\title{
Percutaneous CT-Guided Skull Biopsy: Feasibility, Safety, and Diagnostic Yield
}

\author{
(D) A. Tomasian, (D)T.J. Hillen, and (D).W. Jennings
}

\begin{abstract}
BACKGROUND AND PURPOSE: Although percutaneous musculoskeletal biopsies are routinely performed in the axial and appendicular skeleton, there are no published data on the systematic evaluation of the feasibility, safety, and diagnostic accuracy of percutaneous skull biopsy. In certain clinical encounters such as patients with primary skull tumors or patients with known cancer and isolated skull lesions suspected of calvarial metastasis or synchronous primary tumor, percutaneous skull biopsy may be considered a viable option. The purpose of this study was to evaluate the feasibility, safety profile, and diagnostic yield of percutaneous CT-guided skull biopsy.
\end{abstract}

MATERIALS AND METHODS: Percutaneous CT-guided skull biopsy was performed in 14 patients. Patient demographics, cancer history, indication for initial imaging, imaging technique of diagnosis, skull tumor anatomic location, and final histologic diagnosis were documented. Preprocedural imaging of each skull lesion was reviewed to determine tumor size and characteristics. Procedural notes were reviewed to determine the total conscious sedation time or anesthesia time, type of biopsy needle, and the number and length of obtained core specimens. Procedure-related complications were also documented according to the Society of Interventional Radiology classification.

RESULTS: All CT-guided percutaneous skull biopsy procedures were performed as preoperatively planned and were technically successful. Procedures were performed with the patient under conscious sedation in 93\% (13/14) of cases. Definitive histologic diagnosis was achieved in $86 \%$ (12/14) of cases. There were no acute or delayed procedure-related complications.

CONCLUSIONS: The results of this retrospective initial study suggest that percutaneous CT-guided skull biopsy is feasible with an excellent safety profile, affords a high diagnostic yield for histologic characterization, and may obviate more invasive open skull biopsies.

S kull lesions are uncommonly encountered in clinical practice and are often asymptomatic and discovered incidentally on routine imaging studies acquired for unrelated reasons. Calvarial tumors may also be identified during the work-up of localized symptoms such as pain and palpable masses or defects, as well as on staging studies of other diseases. Primary skull neoplasms comprise approximately $0.8 \%$ of all bone tumors, and although benign lesions are reported to be more common than malignant tumors, the exact prevalence of each has not been established. ${ }^{1}$ Most calvarial lesions have no characteristic imaging features, and correlation of clinical data, particularly a history of cancer, with imaging findings may help direct the diagnostic and therapeutic

Received September 30, 2018; accepted after revision December 8.

From the Department of Radiology (A.T.), University of Southern California, Los Angeles, California; and Mallinckrodt Institute of Radiology (T.J.H., J.W.J.), St. Louis, Missouri.

Please address correspondence to Anderanik Tomasian, MD, Department of Radiology, University of Southern California, 1500 San Pablo St, Los Angeles, CA 90033 e-mail: tomasian.andy@gmail.com

http://dx.doi.org/10.3174/ajnr.A5949 approach. Most clinical oncology protocols and treatment guidelines require that histologic specimens be obtained for proof of diagnosis, as well as genetic and/or molecular testing. Imagingguided percutaneous biopsy of bone lesions has gained wide acceptance as the preferred approach in lieu of open biopsies due to its cost-effectiveness, better safety profile, and high diagnostic accuracy. $^{2-6}$

Although percutaneous biopsies are routinely performed in the axial and appendicular skeleton, there are no published data on the systematic evaluation of the feasibility, safety, and diagnostic accuracy of percutaneous skull biopsy, in part, because there are commonly safer skeletal sites that may be targeted for tissue sampling, particularly in patients with cancer. However, in certain clinical encounters such as patients with primary skull tumors or patients with known cancer and isolated skull lesions suspected of calvarial metastasis or synchronous primary tumor, percutaneous skull biopsy may be considered a viable option.

The purpose of this study was to evaluate the feasibility, safety profile, and diagnostic yield of percutaneous CT-guided skull biopsy.

AJNR Am J Neuroradiol 40:309-12 Feb 2019 www.ajnr.org 

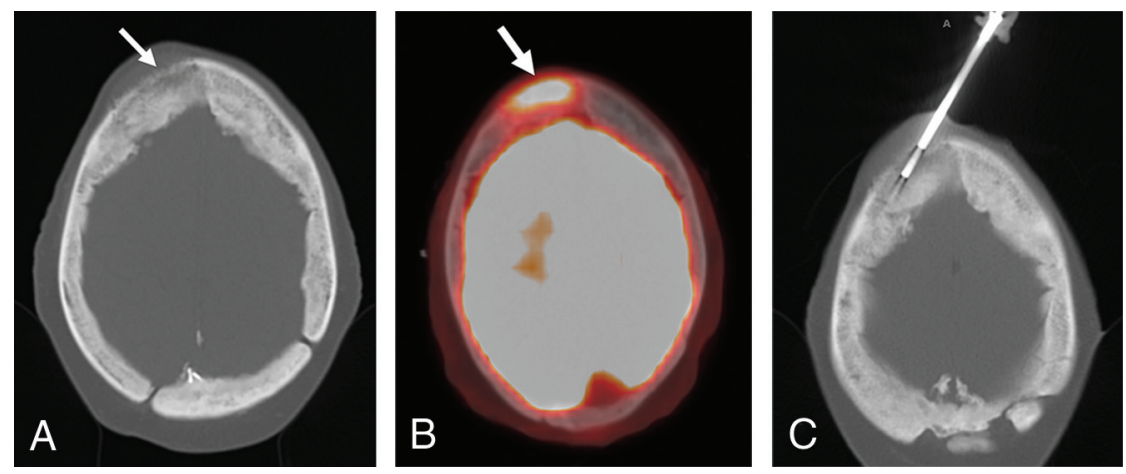

FIG 1. A 58-year-old woman with a history of index finger chondrosarcoma and a right frontal bone mass. Axial CT (A) and FDG-PET/CT (B) images show an aggressive permeative and hypermetabolic bone marrow-replacing lesion in the right frontal bone with an aggressive periosteal reaction ( $A$ and $B$, arrow). Axial CT image during percutaneous biopsy $(C)$ shows positioning of a coaxial bone-biopsy needle within the lesion. The total conscious sedation time was 29 minutes, and histologic evaluation confirmed intraosseous meningioma.
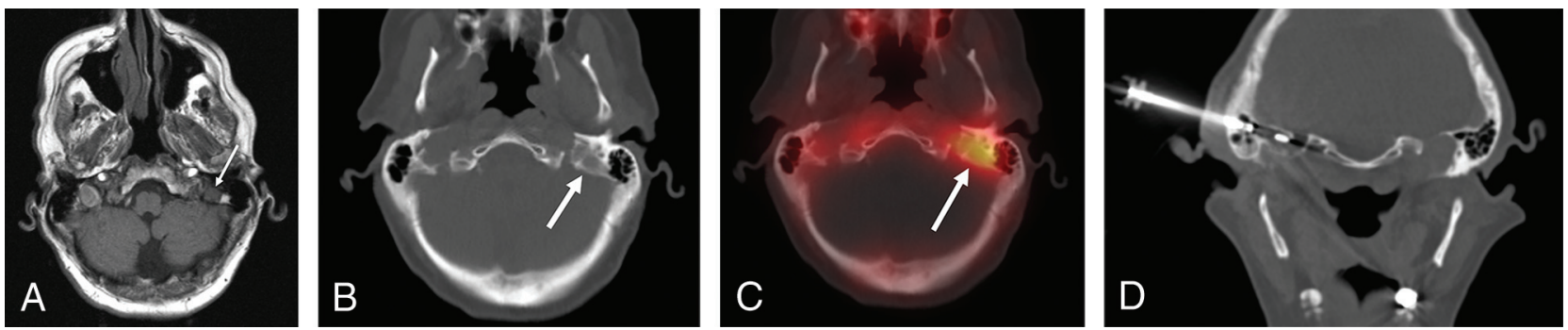

FIG 2. A 65-year-old man with poorly differentiated salivary gland sarcomatoid carcinoma with a left temporal bone lesion. Axial T1-weighted MR imaging (A), CT (B), and technetium 99m methylene diphosphonate SPECT-CT (C) images show an osteolytic bone marrow-replacing lesion with increased radiopharmaceutical uptake in the petrous portion of the left temporal bone $(A-C$, arrow). Note the surrounding vital tissues. Coronal CT image (D) during percutaneous biopsy shows coaxial positioning of a soft-tissue biopsy needle, through an OnControl bone introducer cannula, within the lesion through the mastoid air cells. The total conscious sedation time was 40 minutes, and histologic evaluation confirmed metastasis from a known primary salivary gland tumor.

\section{MATERIALS AND METHODS}

Mallinckrodt Institute of Radiology (IRB) approval was obtained to retrospectively review the institutional data base for all patients who underwent percutaneous CT-guided skull biopsy between 2014 and 2018. Informed consent was waived for retrospective participation in the study. Recorded data included patient demographics, cancer history, indication for initial imaging, imaging technique for diagnosis, skull tumor anatomic location, and final histologic diagnosis. Preprocedural imaging of each skull lesion was reviewed to determine tumor size and characteristics (osteolytic, osteoblastic, or mixed). Procedural notes were reviewed to determine the total conscious sedation or anesthesia time, type of biopsy needle, and the number and length of obtained core specimens.

\section{Biopsy Planning and Procedure}

To ensure safety and minimize the risk of potential complications, the operator must have detailed knowledge of the skull anatomy, and during periprocedural planning and biopsy, attention should be paid to the critical structures, particularly the skull base neurovascular foramina and canals as well as important components of the temporal bone that may be technically more challenging to access and pose higher risks of complications. The authors recommend preprocedural discussions with neurologic surgery or ear, nose, and throat surgery services in all cases to minimize such potential risks.

Preprocedural imaging was reviewed to determine the optimal biopsy approach in all cases. Written informed consent was obtained before all procedures. All procedures were performed using CT guidance with patients either under conscious sedation using midazolam and fentanyl or general anesthesia and were supervised by 2 attending musculoskeletal radiologists, one with $>10$ years of experience and the other with $>8$ years of experience in performing musculoskeletal biopsies. A combination of anatomic landmarks and skin BB markers was used to localize the needle entry site. The implemented measures to reduce the patient radiation dose included decreasing the tube current and gantry rotation time (milliampere-second), decreasing the $\mathrm{x}$-ray beam energy (kilovolt [peak]), increasing the pitch (at a set tube current), central patient positioning at the $\mathrm{CT}$ isocenter, and limiting the scan length to the volume of interest. Following the initial guidance scan, which was performed with standard-dose CT parameters for optimal assessment of the ROI, a reduced CT dose protocol was used for the subsequent scans, which included $100 \mathrm{mAs}, 100$ $\mathrm{kV}(\mathrm{p})$, and pitch of $1.0-1.2$, with a $2-\mathrm{mm}$ section thickness. Both bone and soft-tissue CT windows were used for needle guidance. Superficial and deep soft-tissue and periosteal anesthesia were achieved with $50: 50$ dilution of $1 \%$ lidocaine and $0.25 \%$ bupivacaine.

The procedures were performed using 1 of 2 coaxial biopsy needle systems, each with a $6-\mathrm{cm}$ introducer cannula and a $10-\mathrm{cm}$ biopsy needle: 1) a coaxial battery-powered hand drill with a 10 (11)-ga introducer cannula and 12 (13)-ga inner diamond-tipped needle (Arrow OnControl; Vidacare, Shavano Park, Texas). The 
inner needle is then exchanged for a 12(13)-ga hollow biopsy needle. This needle system was used in $86 \%(12 / 14)$ of cases; or 2$)$ a coaxial soft-tissue core biopsy system with a13-ga introducer cannula and 14-ga spring-loaded biopsy needle (Bard Marquee, C.R. Bard, Tempe, Arizona; or Achieve, Merit Medical, South Jordan, Utah) was used. The soft-tissue core biopsy system was used if the lesion was entirely osteolytic with extensive cortical destruction or a soft-tissue component. In addition, in cases in which lesion access was achieved with the bone core biopsy system and no specimen could be obtained due to the destructive nature of the mass, a hollow soft-tissue core biopsy needle was placed coaxially through the existing bone core introducer cannula to obtain tissue (Fig 1 and 2).

Contraindications to imaging-guided skull biopsies included percutaneously inaccessible lesions, coagulopathy (platelet count of $<50,000$ per microliter and an international normalized ratio of $>1.5$ ), and allergy to conscious sedation or general anesthesia medications.

\section{Outcome Assessment}

Procedural complications were documented according to Society of Interventional Radiology classification. ${ }^{7}$ Patients were clinically monitored for a minimum of 2 hours after each procedure for evidence of acute complications, such as hematoma formation or neurologic injury. A follow-up phone call was performed 24 hours following the biopsy by a nurse coordinator, and electronic medical records were also reviewed for evidence of delayed complications within 30 days of the biopsy. Procedures were considered technically successful if the biopsy needle was visualized within the target lesion and core specimens were obtained. Surgical pathology reports were reviewed to assess the diagnostic yield. Negative biopsy results were not pursued further when follow-up imaging confirmed lesion stability or a negative result was considered concordant with a low pretest probability of an aggressive etiology. Surgical pathology reports were also reviewed for the presence of crush artifacts. If crush artifacts were present, the specimen was considered histologically adequate if it could still be used to confirm or exclude the diagnosis in question.

\section{RESULTS}

All biopsy procedures were performed as preoperatively planned and were technically successful. According to the Society of Interventional Radiology classification, there were no acute or delayed procedure-related complications. Percutaneous CT-guided skull biopsy was performed on 14 patients (men, $n=5$, and women, $n=9)$ with an age range of 35-84 years. Fifty-seven percent $(8 / 14$ patients) of patients had a history of cancer. Indications for imaging included oncologic staging (57\%, 8/14 patients), palpable mass (7\%, 1/14 patients), cranial nerve dysfunction (7\%, 1/14 patients), and imaging for unrelated reasons (incidental diagnosis) $(29 \%, 4 / 14$ patients). Preprocedural imaging included PET/CT $(n=4)$, MR imaging $(n=7)$, CT $(n=9)$, and bone scintigraphy $(n=3)$, including SPECT $(n=1)$. Procedures were performed with the patient under conscious sedation in $93 \%$ (13/ 14) of cases and under general anesthesia in 7\% (1/14) of cases. The median conscious sedation duration was 40 minutes (range,
27-75 minutes). The median midazolam and fentanyl doses administered were $2.5 \mathrm{mg}$ (range, $1-4 \mathrm{mg}$ ) and $150 \mu \mathrm{g}$ (range, 75-300 $\mu \mathrm{g}$ ), respectively. The total anesthesia time was 30 minutes for procedures performed with the patient under general anesthesia. Definitive histologic diagnosis was achieved in 86\% (12/14) of cases.

There was no biopsy specimen with crush artifacts. In 1 patient with a palpable left frontal bone mass that was characterized on MR imaging, histologic analysis showed blood products with benign bone and soft tissue that were considered concordant with imaging findings suggestive of hemangioma. In 1 patient with a left frontal bone osteolytic lesion incidentally identified on head CT and subsequently characterized on bone scintigraphy demonstrating increased radiopharmaceutical uptake, histologic analysis showed no evidence of malignancy with benign bone and soft tissue. The lesion remained unchanged on 2-year follow-up imaging; this finding was considered concordant with the biopsy results. The details of the patient cohort including skull lesion anatomic location, tumor size, tumor characteristics, type of biopsy needle, number and length of obtained core specimens, and final histologic diagnosis are shown in the Table.

\section{DISCUSSION}

The results of the present study demonstrate that percutaneous CT-guided skull biopsy is feasible and safe with a high diagnostic yield. Although percutaneous imaging-guided biopsy of musculoskeletal lesions, including appendicular and spinal bone tumors, has been well-established and accepted as a safe and costeffective approach with high diagnostic accuracy for histologic characterization, ${ }^{2-6}$ to our knowledge, there is essentially no published literature to date on the systematic evaluation of the safety profile and diagnostic yield of percutaneous imaging-guided skull biopsy. This is, in part, because there are often other skeletal lesions that are safer and less technically challenging to target for biopsy, particularly in oncologic patients. However, with progressively increasing use of routine cross-sectional imaging and oncologic staging imaging in clinical practice, isolated skull lesions in patients with or without a history of cancer may be identified more frequently, and percutaneous tissue sampling of a calvarial lesion may be the only minimally invasive viable option to both establish a new diagnosis such as new or synchronous primary tumor or confirm metastasis. Percutaneous imaging-guided skull biopsy poses a unique challenge due to the proximity of brain tissue, critical facial structures, and vital neurovascular structures in the skull base, which may discourage both clinicians and radiologists from requesting and performing such procedures, respectively.

In this initial experience, all procedures were technically successful with excellent safety profiles, characterized by lack of procedure-related complications according to the Society of Interventional Radiology classification. ${ }^{7}$ The median conscious sedation duration was 40 minutes (range, $27-75$ minutes), which is within the reasonable range for imaging-guided percutaneous bone biopsies. ${ }^{5,6}$ Diagnostic yield refers to the likelihood that a test or procedure will provide the information needed to establish a diagnosis and is defined as diagnostic results divided by the sum of diagnostic results and nondiagnostic results. Therefore, diagnostic yield is the most direct and accurate representation of the biopsy success rate. In our study, definitive histologic diagnosis 
Details of skull lesions, biopsy parameters, and final diagnosis ${ }^{a}$

\begin{tabular}{|c|c|c|c|c|c|c|c|}
\hline Patient No. & Anatomic Site & $\begin{array}{l}\text { Tumor Size } \\
(\mathrm{mm})\end{array}$ & $\begin{array}{l}\text { Tumor } \\
\text { Feature }\end{array}$ & $\begin{array}{l}\text { Biopsy } \\
\text { Needle }\end{array}$ & $\begin{array}{l}\text { Specimen } \\
\text { No. }\end{array}$ & $\begin{array}{l}\text { Core Length } \\
(\mathrm{mm})\end{array}$ & Final Diagnosis \\
\hline 1 & Right frontal & $50 \times 46 \times 22$ & Permeative & Bone & 3 & 20 & Intraosseous meningioma \\
\hline \multirow[t]{2}{*}{2} & \multirow[t]{2}{*}{ Left frontal } & \multirow[t]{2}{*}{$13 \times 9 \times 5$} & \multirow[t]{2}{*}{ Osteolytic } & Bone & 1 & 10 & \multirow{2}{*}{$\begin{array}{l}\text { Blood products and benign } \\
\text { tissue }\end{array}$} \\
\hline & & & & Soft tissue & 2 & 10 & \\
\hline 3 & Left frontal & $61 \times 42 \times 13$ & Osteoblastic & Bone & 7 & $2-10$ & B-cell lymphoma \\
\hline \multirow[t]{2}{*}{4} & \multirow[t]{2}{*}{ Right frontal } & \multirow[t]{2}{*}{$9 \times 9 \times 5$} & \multirow[t]{2}{*}{ Osteolytic } & Bone & 1 & 6 & \multirow[t]{2}{*}{ Hemangioma } \\
\hline & & & & Soft tissue & 1 & 5 & \\
\hline \multirow[t]{2}{*}{5} & \multirow[t]{2}{*}{ Left frontal } & \multirow[t]{2}{*}{$11 \times 10 \times 4$} & \multirow[t]{2}{*}{ Osteolytic } & Bone & 1 & 5 & \multirow{2}{*}{$\begin{array}{l}\text { Benign bone and soft } \\
\text { tissue }\end{array}$} \\
\hline & & & & Soft tissue & 2 & 10 & \\
\hline 6 & Skull base and occipital & Diffuse & Mixed & Bone, soft tissue & 6 & $15-20$ & Fibrous dysplasia \\
\hline \multirow[t]{2}{*}{7} & \multirow[t]{2}{*}{ Left temporal } & \multirow[t]{2}{*}{$19 \times 14 \times 10$} & \multirow[t]{2}{*}{ Osteolytic } & Bone & 1 & 15 & \multirow{2}{*}{$\begin{array}{l}\text { Metastatic salivary } \\
\text { sarcomatoid carcinoma }\end{array}$} \\
\hline & & & & Soft tissue & 1 & 20 & \\
\hline 8 & Right temporal & $21 \times 15 \times 7$ & Osteolytic & Soft tissue & 5 & $10-20$ & Plasmocytoma \\
\hline \multirow[t]{2}{*}{9} & \multirow[t]{2}{*}{ Left parietal } & \multirow[t]{2}{*}{$38 \times 34 \times 10$} & \multirow[t]{2}{*}{ Mixed } & Bone & 1 & 10 & \multirow[t]{2}{*}{ Metastatic breast carcinoma } \\
\hline & & & & Soft tissue & 1 & 20 & \\
\hline 10 & Right occipital & $12 \times 8 \times 8$ & Osteolytic & Bone & 1 & 20 & Osteonecrosis \\
\hline 11 & Left occipital & $27 \times 20 \times 19$ & Osteolytic & Soft tissue & 2 & 20 & Metastatic thyroid carcinoma \\
\hline 12 & Right parietal & $24 \times 18 \times 13$ & Mixed & Bone & 2 & $4-6$ & Metastatic breast carcinoma \\
\hline 13 & Left parietal & $\begin{array}{c}\text { Most of parietal } \\
\text { bone }\end{array}$ & Permeative & $\begin{array}{l}\text { Bone } \\
\text { Soft tissue }\end{array}$ & $\begin{array}{l}1 \\
6\end{array}$ & $\begin{array}{c}5 \\
10-20\end{array}$ & Radiation-induced sarcoma \\
\hline 14 & Left frontal & $27 \times 17 \times 14$ & Osteoblastic & Bone & 2 & 15 & Xanthoma with osteosclerosis \\
\hline
\end{tabular}

${ }^{a}$ Data for patients 3 and 6 have been previously published. ${ }^{8}$

was achieved in $86 \%(12 / 14)$ of patients, which directly influenced patients' management by confirming metastasis, new primary tumor, synchronous primary tumor (patient 3), or benign histology (Table). In 2 patients with no definitive histologic diagnosis, biopsy results were considered concordant with imaging features indicating a benign etiology.

The authors used a coaxial bone-biopsy needle system with a battery-powered hand drill in $86 \%(12 / 14)$ of patients (with or without a soft-tissue biopsy needle), which facilitates needle purchase and advancement within the bone by providing the operator greater control over the needle trajectory via recruitment of wrist and hand muscles with more fine-movement capabilities compared with standard manual techniques in which direct pushing and rotation of the biopsy needle knob or handle are implemented and proximal arm and shoulder musculature are used. This system is particularly important in skull biopsies because it results in more accurate targeting of lesions and avoidance of injury to surrounding critical structures. In 2 patients, due to the extensive destructive nature of the target lesions, soft-tissue needles were used to perform the biopsy. In addition, if a skull lesion is accessed with a coaxial bone-biopsy system and the initial biopsy attempt results in no specimen, which is not unusual in destructive osteolytic lesions, the authors recommend coaxial placement of a soft-tissue biopsy needle through the existing bone biopsy introducer cannula for improved sample yield. In 2015, Wallace et al ${ }^{8}$ reported 2 cases of successful percutaneous CT-guided skull biopsy confirming histologic diagnoses with no complications, and the authors suggested the feasibility and safety of the procedure.

The major limitations of the present study include the retrospective methodology and the small number in the patient cohort.

\section{CONCLUSIONS}

The results of this retrospective initial study suggest that percutaneous CT-guided skull biopsy is feasible with an excellent safety profile and affords a high diagnostic yield for histologic characterization and may obviate more invasive open-skull biopsies.

Disclosures: Travis J. Hillen-UNRELATED: Consultancy: Medtronic. Jack W. Jennings-RELATED: Consulting Fee or Honorarium: Bard, Comments: The consulting was for a device that has not yet gone to market; UNRELATED: Consultancy: Merit, BTG, Medtronic; Payment for Lectures Including Service on Speakers Bureaus: Merit, payment made to individual.

\section{REFERENCES}

1. Shah MV, Haines SJ. Pediatric skull, skull base, and meningeal tumors. Neurosurg Clin N Am 1992;3:893-924 CrossRef Medline

2. Skrzynski MC, Biermann JS, Montag A, et al. Diagnostic accuracy and charge-savings of outpatient core needle biopsy compared with open biopsy of musculoskeletal tumors. J Bone Joint Surg Am 1996;78: 644-49 CrossRef Medline

3. Fraser-Hill MA, Renfrew DL. Percutaneous needle biopsy of musculoskeletal lesions, 1: effective accuracy and diagnostic utility. AJR Am J Roentgenol 1992;158:809-12 CrossRef Medline

4. Fraser-Hill MA, Renfrew DL, Hilsenrath PE. Percutaneous needle biopsy of musculoskeletal lesions, 2: cost-effectiveness. AJR Am J Roentgenol 1992;158:813-18 CrossRef Medline

5. Traina F, Errani C, Toscano AJ, et al. Current concepts in the biopsy of musculoskeletal tumors: AAOS exhibit selection. Bone Joint Surg Am 2015;97:e7 CrossRef Medline

6. Wallace AN, McWilliams SR, Wallace A, et al. Drill-assisted biopsy of the axial and appendicular skeleton: safety, technical success, and diagnostic efficacy. J Vasc Interv Radiol 2016;27:1618-22 CrossRef Medline

7. Ahmed M, Solbiati L, Brace CL, et al; International Working Group on Image-Guided Tumor Ablation, Interventional Oncology Sans Frontières Expert Panel, Technology Assessment Committee of the Society of Interventional Radiology, Standard of Practice Committee of the Cardiovascular and Interventional Radiological Society of Europe. Image-guided tumor ablation: standardization of terminology and reporting criteria: a 10-year update. J Vasc Interv Radiol 2014;25:1691705 CrossRef Medline

8. Wallace AN, Tomasian A, Hsi AC, et al. CT-guided percutaneous skull biopsy using a drill-assisted system: technical report of two cases. Interv Neuroradiol 2015;21:774-79 CrossRef Medline 